\title{
Intestinal behavior of the ester prodrug tenofovir DF in humans
}

Sophie Geboers ${ }^{1}$, Steven Haenen ${ }^{1}$, Raf Mols ${ }^{1}$, Joachim Brouwers ${ }^{1}$, Jan Tack ${ }^{2}$, Pieter Annaert ${ }^{1}$, Patrick Augustijns $^{1 a}$

${ }^{1}$ Drug Delivery and Disposition, KU Leuven, Department of Pharmaceutical and Pharmacological Sciences, Belgium

${ }^{a}$ Corresponding author. Drug Delivery and Disposition, KU Leuven Department of Pharmaceutical and Pharmacological Sciences, Gasthuisberg O\&N 2, Herestraat 49 Box 921, 3000 Leuven, Belgium. Tel.: +32 16 330301; fax: +32 16 330305; patrick.augustijns@pharm.kuleuven.be

${ }^{2}$ University Hospitals Leuven, Department of Gastroenterology, Belgium

Tenofovir, ester prodrug, intestinal absorption, human intestinal fluids, clinical trial 


\section{ABSTRACT}

Tenofovir-disoproxil-fumarate (TDF) is a double ester prodrug which enables intestinal uptake of tenofovir (TFV) after oral administration in humans. In this study, prodrug stability was monitored in situ in the human intestine and in vitro using biorelevant media. In fasted state human intestinal fluids, the prodrug was completely degraded within $90 \mathrm{~min}$, resulting in the formation of the mono-ester intermediate and TFV; in fed state intestinal fluids, the degradation rate of TDF was reduced and no TFV was formed. Intestinal fluid samples aspirated after administration of TDF confirmed extensive intraluminal degradation of TDF in fasted state conditions; a relatively fast absorption of TDF partly compensated for the degradation. Although food intake reduced intestinal degradation, the systemic exposure was not proportionally increased. The lower degradation in fed state conditions may be attributed to competing esterase substrates present in food, lower chemical degradation in the slightly more acidic environment and micellar entrapment, delaying exposure to the "degrading" intestinal environment. The results of this study demonstrate premature intestinal degradation of TDF and suggest that TFV may benefit from a more stable prodrug approach; however, fast absorption may compensate for fast degradation, indicating that prodrug selection should not be limited to stability assays.

\section{INTRODUCTION}

Tenofovir (TFV) is a nucleotide reverse transcriptase inhibitor which is used for the treatment of hepatitis $B$ and HIV-infections. It is a very hydrophilic compound $\left(\log D_{7.4}=-3.55\right)$ containing two negative charges resulting in low intestinal permeability; therefore, it is classified as a BCS class III compound. (Kearney et al., 2004) One of the strategies used to overcome permeability issues is to increase the lipophilicity of the compound through a prodrug strategy. A prodrug is an inactive form of the drug, which has to be activated once taken up. (Rautio et al., 2008) At this moment, TFV is on the market as the bis-ester prodrug tenofovir disoproxil fumarate (TDF, Viread $\left.{ }^{\circledR}\right)$. In this prodrug, two isopropyloxycarbonyloxymethyl moieties mask the negative charges of TFV, to form a lipophilic ester prodrug that results in an increased transport across the intestinal epithelium. The prodrug is hydrolyzed in two steps: first, TDF is hydrolyzed 
by carboxylesterases to the mono-ester intermediate; this intermediate is further hydrolyzed by phosphodiesterases to the active form TFV. (figure 1) These enzymes are present in the lumen of the intestine, coming from the secretion of the pancreas and from the enterocytes. Only the active form is observed in the systemic circulation. (Fardis and Oliyai, 2007) Compared to direct oral administration of TFV, intake of the prodrug results in a bioavailability increase of tenofovir from $2 \%$ to $20 \%$ in mice and from $17.1 \%$ to $30.1 \%$ in dogs. (Naesens et al., 1998); (Cundy et al., 1998) Although the bioavailability is increased, it remains relatively low. Premature degradation of the ester prodrug in the intestinal tract, either by chemical or by enzymatic hydrolysis, might contribute to the limited efficiency of the prodrug approach.

The intestinal absorption behavior of TDF has been studied in different experimental set-ups including Caco-2 cells, in situ perfusion systems and cellular incubation systems. (Barditch-crovo et al., 2001); (Heimbach et al., 2003); (Roux et al., 2013); (Van Gelder et al., 1999) However, to the best of our knowledge, no in vivo data about the intestinal behavior of TDF has been collected yet. When reviewing the available in vitro data about the decomposition of TDF, contradictory observations have been reported. Roux et. al. found that decomposition of TDF resulted predominantly from chemical degradation: the decomposition rate $\left(k_{c}\right)$ amounted to $6.6 \times 10^{-3} \mathrm{~min}^{-1}$ for the chemical degradation as compared to $1.1 \times 10^{-3} \mathrm{~min}^{-1}$ for the enzymatic degradation (Roux et al., 2013). Lung-Chi et. al. further explored the influence of $\mathrm{pH}$ on the degradation kinetics of ester prodrugs containing an oxycarbonyloxymethyl group (including TDF) and concluded that these prodrugs are most stable in a $\mathrm{pH}$ range from 2 to 3 . When increasing the $\mathrm{pH}$ above 3, they become exponentially more sensitive to chemical hydrolysis. (Lung-Chi et al., 2001)

In contrast, Van Gelder et al. attributed TDF degradation during absorption primarily to the enzymatic pathway. By using homogenates of Caco-2 cells and intestinal homogenates from rat, pig and humans, a very fast esterase-mediated degradation of the ester-prodrug was observed. The role of esterases was confirmed by the fact that the degradation was inhibited by co-incubation with esterase inhibitors; 
degradation was also decreased upon co-incubation with fruit extracts, suggesting competitive inhibition by the esters present in these extracts. (Van Gelder et al., 2002)

The data so far suggest that chemical as well as enzymatic degradation may lead to a premature decomposition of TDF in the intestinal tract, resulting in a decreased availability of the prodrug and, eventually, reduced absorption. A drawback of the available literature data is that they provide at best indirect evidence of the intraluminal behavior of TDF in humans. We therefore decided to directly explore the intraluminal behavior of TDF by collecting and characterizing intestinal fluids from healthy volunteers after oral intake of TDF, in both fasting and fed condition. As demonstrated for other drugs, the parallel assessment of intraluminal and systemic concentrations may allow linking the intestinal behavior to the bioavailability of the drug. (Brouwers et al., 2007); (Brouwers and Augustijns, 2014) The specific aims of this study were (1) to investigate the in vitro degradation profile of TDF in fasted and fed state human intestinal fluids (FaHIF, FeHIF) in order to assess the risk for intestinal decomposition of ester prodrugs prior to absorption, (2) to explore the intraluminal behavior of TDF in healthy volunteers by collecting intestinal fluids after oral intake of TDF and (3) to investigate possible food effects on the intraluminal behavior of TDF. 


\section{MATERIALS AND METHODS}

\subsection{Chemicals}

TDF was provided by the NIH AIDS Research and Reference Reagent Program (Germantown, MD). TFV was obtained from Watson International (Kunshan City, China). Dimethylsulfoxide (DMSO) and tetrabutylammonium sulfate were obtained from Acros-Organics (Geel, Belgium). Acetic acid was obtained from Chem-lab (Zedelgem, Belgium). Acetonitrile was purchased from Fisher Scientific (Leicestershire, UK). Monobasic potassium phosphate monohydrate $\left(\mathrm{KH}_{2} \mathrm{PO}_{4} \cdot \mathrm{H}_{2} \mathrm{O}\right)$, bis-4nitrophenylphosphate, chloroacetaldehyde and pancreatin from porcine pancreas (powder, suitable for cell culture, 4× USP specifications) were obtained from Sigma-Aldrich (St. Louis, MO). Methanol and sodium acetate were purchased from VWR International (Leuven, Belgium). Water was purified with a Maxima system (Elga Ltd., High Wycombe Bucks, UK). All stock solutions were prepared in dimethyl sulfoxide. The mono-ester intermediate of TDF was obtained by degradation of TDF at neutral $\mathrm{pH}$ : a $100 \mu \mathrm{M}$ solution of TDF was made in a phosphate buffer $(\mathrm{pH} 7)$; following chemical degradation during $7 \mathrm{~h}$ at $60^{\circ} \mathrm{C}$, an equal amount of $1 \mathrm{M} \mathrm{HCl}$ was added to the solution; quantitative transformation into the intermediate without formation of TFV was confirmed.

\subsection{Stabilization mixture}

All samples taken during the experiments were diluted in a stabilization mixture to ensure the stability of the ester prodrug and mono-ester intermediate during further processing. The stabilization mixture consisted of $\mathrm{MeOH}: 0.02 \mathrm{~N} \mathrm{HCl}$ (50:50) containing $400 \mu \mathrm{M}$ of the esterase inhibitor bisnitrophenylphosphate.

\subsection{In vitro stability studies}

\subsubsection{Simulated intestinal fluids}

The in vitro degradation of TDF was investigated in mixtures of FaSSIF and FeSSIF containing 10 mg pancreatin/mL. The inclusion of pancreatin in FaSSIF has been reported by Borde et al. and the United States Pharmacopoeia 2009 to explore the degradation of ester prodrugs. (Borde et al., 2012); (“U.S. 
Pharmacopeial Convention," 2009) FaSSIF and FeSSIF were made according to the manufacturer's

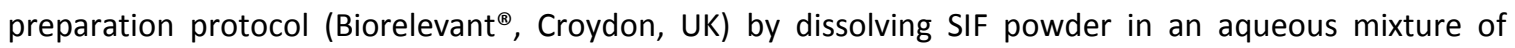
$\mathrm{NaOH} / \mathrm{NaH}_{2} \mathrm{PO}_{4} / \mathrm{NaCl}$ for FaSSIF and $\mathrm{NaOH} / \mathrm{CH}_{3} \mathrm{COOH} / \mathrm{NaCl}$ for FeSSIF. The pH was adjusted to 6.5 for FaSSIF and to 5.0 for FeSSIF. After the addition of pancreatin, the solutions were centrifuged and the supernatant was used for stability studies. TDF (stock solution of $5 \mathrm{mM}$ in DMSO) was spiked into $1 \mathrm{~mL}$ of FaSSIF and FeSSIF resulting in a start concentration of $50 \mu \mathrm{M}$. Samples were taken at predetermined time points and immediately diluted 100 times in the stabilization mixture. The diluted samples were centrifuged and the supernatant was used for analysis.

\subsubsection{Human intestinal fluids}

Fasted state human intestinal fluids (FaHIF) and fed state human intestinal fluids (FeHIF) were aspirated from four healthy volunteers (two males, two females, between 23 and 27 years old). The study was approved by the Committee of Medical Ethics of the University Hospitals Leuven, Belgium and the procedure followed the tenets of the Declaration of Helsinki. HIF were collected from the duodenum (D2D3) with a double-lumen polyvinyl catheter according to a previously described protocol. (Clarysse et al., 2011) Samples were collected every $10 \mathrm{~min}$ for $120 \mathrm{~min}$ in the fasted state and for $90 \mathrm{~min}$ in the fed state. In the fed state, the nutritional drink Ensure Plus ${ }^{\circledR}$ was given to the volunteers. Pooled samples were made for each nutritional state, by combining equal volumes of the aspirates from all four volunteers. Pooled HIF were stored at $-26^{\circ} \mathrm{C}$ until further use. The $\mathrm{pH}$ amounted to 7.6 for the fasted state pool and to 5.5 for the fed state pool.

For the stability studies, a DMSO stock solution of TDF $(5 \mathrm{mM})$ was spiked into $1 \mathrm{~mL}$ of FaHIF/FeHIF generating a concentration of $50 \mu \mathrm{M}$; this solution was incubated at $37^{\circ} \mathrm{C}$ for $1 \mathrm{~h}$. Every $15 \mathrm{~min}$, a sample was taken and immediately diluted 100 times in the stabilization mixture. These samples were centrifuged and the supernatant was used for analysis. During the stability studies, the $\mathrm{pH}$ was measured using a Portamess 911 pH-meter (Knick GmbH \& Company, Berlin, Germany).

2.4 Clinical study 
To study the intraluminal behavior of TDF in the fasted and the fed state in humans, a crossover study was performed in five healthy volunteers (aged between 22 and 26 years; two men and three women). The procedure followed the tenets of the Declaration of Helsinki and was approved by the Committee of Medical Ethics of the University Hospitals Leuven, Belgium. All volunteers provided written informed consent to participate in this study. After an overnight fast ( $>12 \mathrm{~h}$ ), one double-lumen polyvinyl catheter [(Salem Sump Tube $14 \mathrm{Ch}$ (external diameter $4.7 \mathrm{~mm}$ ), Sherwood Medical, Petit Rechain, Belgium] was introduced via the nose and positioned into the duodenum (D2/D3). The position of the tube was checked by means of fluoroscopy. It has previously been reported that the presence of a transpyloric tube does not influence gastric emptying or duodenogastric reflux. (Müller-Lissner et al., 1982) For the experiments in the fasted state, a single tablet of Viread ${ }^{\circledR}$ (300mg of TDF) was administered with $250 \mathrm{ml}$ of water. For the experiments in the fed state, $400 \mathrm{ml}$ of a nutritional drink (Fortimel ${ }^{\circledR}$ ) was given 20 min prior to intake of the TDF tablet. Fortimel (Nutricia, Strombeek-Bever, Belgium) was used to simulate a standard meal. One portion of $400 \mathrm{~mL}$ has an energy content of $2520 \mathrm{~kJ}$, of which lipids, carbohydrates, and proteins constitute $32 \%, 41 \%$, and $27 \%$ on energy basis, respectively; the osmolality amounts to $470 \mathrm{mOsm} / \mathrm{kg}$; the $\mathrm{pH}$ is 6.8. Volunteers were asked to sit in upright position in a bed during the sampling procedure. Samples of human intestinal fluid (sample volume between 1.5 and $4 \mathrm{ml}$ ) were aspirated every 15 min up to $4 \mathrm{~h}$ for both states. In parallel to the sampling of gastrointestinal fluids, venous blood samples were collected in heparinized tubes (BD Vacutainer systems, Plymouth, UK) at 0, 20, 40, 60, 80, 100, 120, 160, $180,210,240,300,360,420$ and 480 min after drug intake. These blood samples were centrifuged at $1,699 \times \mathrm{g}$ for $10 \mathrm{~min}$ to gain plasma samples which were stored at $-26^{\circ} \mathrm{C}$ until further analysis. The intestinal samples were stabilized directly after collection by a 100 -fold dilution in stabilization mixture (see section 2.2). After stabilization, the samples were stored at $-26^{\circ} \mathrm{C}$ until further analysis.

\subsection{Analysis of TFV}

For the analysis of TFV, both plasma and intestinal samples were processed to obtain a fluorescent derivate of TFV. This derivatization was performed based on a previously described method. (Mallants et al., 2005); (Sparidans et al., 2003) In 200 $\mathrm{L}$ samples of plasma or stabilized dilutions of intestinal aspirates, 
proteins were precipitated by adding $600 \mu \mathrm{l}$ of methanol followed by vortexing (30 s). Supernatant obtained after centrifugation $(20,817 \times \mathrm{g}, 10 \mathrm{~min})$ was transferred to new test tubes and evaporated under a gentle stream of air. The residue was redissolved in $200 \mu \mathrm{l}$ of a buffer solution consisting of $100 \mathrm{mM}$ sodium acetate $(\mathrm{pH} 4.5)$ and $3.0 \%$ of the derivatizing agent chloroacetaldehyde. This solution was vortexed and kept at $80^{\circ} \mathrm{C}$ for $50 \mathrm{~min}$. In this way, tenofovir was converted into its fluorescent derivative (Naesens et al., 1992). The samples were cooled to $-30^{\circ} \mathrm{C}$ for $5 \mathrm{~min}$. After centrifugation at $20,817 \times \mathrm{g}$ for 10 min, supernatant was collected and transferred into microvials to be analyzed by HPLC.

After derivatization of the intestinal and plasma samples, concentrations of the fluorescent derivative of TFV were measured by reversed-phase HPLC and fluorescence detection. (Mallants et al., 2005) A volume of $30 \mu \mathrm{l}$ was injected into a Waters HPLC system consisting of a $600 \mathrm{E}$ controller and pump, a 717plus autosampler and a Novapak C-18 column under radial compression (Waters, Milford, MA). Fluorescence signals (excitation $260 \mathrm{~nm}$, emission $425 \mathrm{~nm}$ ) were monitored with a Jasco FP-1520 fluorescence detector (Tokyo, Japan). Data acquisition and integration were performed using Empower Pro (Empower 2) (Waters, Belgium) software. The mobile phase consisted of a phosphate buffer (10 mM KH $\mathrm{PO}_{4}, 2 \mathrm{mM}$ tetrabutylammonium sulfate, $\mathrm{pH} 8.0$ ) (A) and acetonitrile (B). The flow rate amounted to $1.0 \mathrm{~mL} / \mathrm{min}$. Separation was carried out with a gradient elution starting with $75 \%$ A and $25 \%$ B for 3.5 min, followed by a linear gradient from $25 \%$ to $90 \%$ B over 0.5 min, which was accompanied with a change in flow rate from $1 \mathrm{~mL} / \mathrm{min}$ to $1.5 \mathrm{~mL} / \mathrm{min}$. After $7 \mathrm{~min}$, the mobile phase was changed back to its initial conditions and the column was re-equilibrated during $7 \mathrm{~min}$. The retention time of derivatised TFV was $4.5 \mathrm{~min}$. Calibration curves in plasma and HIF were found to be linear in a concentration range of $0.03 \mu \mathrm{M}-1 \mu \mathrm{M}$. The repeatability and precision were tested for the plasma and HIF samples at concentrations of $1 \mu \mathrm{M}$ and $0.05 \mu \mathrm{M}$, respectively. For both concentrations, the mean bias and variability were $<10 \%$.

\subsection{Analysis of TDF and mono-ester}

Concentrations of TDF and its mono-ester intermediate in diluted intestinal samples were determined using HPLC with MS/MS detection. The Thermo Scientific (San Jose, USA) LCQ Deca XP Max iontrap mass 
spectrometer system equipped with an electrospray ionization (ESI) source was used. Data acquisition and peak integration were performed with Xcalibur $^{\circledR}$. A Kinetex ${ }^{\circledR}$ XB-C18 column (length $50 \mathrm{~mm} \times 2.1 \mathrm{~mm}$, particle size 2.6 $\mu \mathrm{m}$ ) (Phenomenex, The Netherlands), protected by a Krudkatcher Ultra HPLC In-Line filter (Phenomenex), was used for chromatographic separation. The mobile phases consisted of $0.2 \%$ formic acid (A), $5 \mathrm{mM}$ ammonium acetate buffer $(\mathrm{pH} 4.8)$ (B) and acetonitrile (C). The flow rate amounted to 200 $\mu \mathrm{L} / \mathrm{min}$. Separation was carried out with a gradient elution starting with $81 \%$ A, $9 \%$ B and $10 \%$, changing to $55.5 \% \mathrm{~A}, 10.5 \% \mathrm{~B}, 34 \% \mathrm{C}$ after $1 \mathrm{~min}$. After $2 \mathrm{~min}$ the composition changed immediately to $30 \% \mathrm{~A}, 10 \%$ B, $60 \%$ C. After $2.5 \mathrm{~min}$, the column was re-equilibrated with the initial conditions for $3 \mathrm{~min}$. The total run time was $8.5 \mathrm{~min}$ and the injection volume amounted to $20 \mu \mathrm{L}$ (full loop mode). The mass spectrometer was operated in the positive electrospray (ESI) mode for TDF and in the negative ESI mode for the monoester. The spray voltage, capillary voltage and capillary temperature were $4.50 \mathrm{kV}, 12 \mathrm{~V}$ and $325^{\circ} \mathrm{C}$, respectively. Nitrogen was used as the sheath gas (59 arbitrary units), ion sweep and auxillary gas (20 arbitrary units). Argon was used as the collision gas at a pressure of $1.5 \mathrm{mTorr}$. The mass spectrometer was operated in the selected reaction monitoring (SRM) mode. A single precursor-product ion pair was used for detection: $\mathrm{m} / \mathrm{z}_{\mathrm{TDF}} 520.20 \rightarrow 288.0$ (collision energy: $32 \mathrm{~V}$ ) and $\mathrm{m} / \mathrm{z}_{\text {Mono }} 402.10 \rightarrow 328.00$ (collision energy: $32 \mathrm{~V}$ ), both with a scan time of $100 \mathrm{~ms}$. Calibration curves were linear over the concentration range of 0.03 to $1 \mu \mathrm{M}$. The repeatability and precision for TDF and its mono-ester intermediate were assessed at a concentration of $0.3 \mu \mathrm{M}$. Repeatability and precision errors were found to be $<10 \%$ for both compounds.

\subsection{Data presentation and Statistical Analysis}

All concentration-time profiles obtained from the in vitro studies are presented as mean \pm sd for three experiments. All concentration-time profiles from the clinical trial are presented as mean \pm sem for five subjects. A paired t-test was used to compare selected parameters ( $A \cup C_{0-4 h}$ and $A U C_{0-8 h}$ ) of the in vivo concentration-time profiles between the fasted and fed state condition. When $p<0.05$, differences were considered statistically significant. 


\section{RESULTS AND DISCUSSION}

The in vitro Caco-2 system as well as the rat in situ intestinal perfusion setup have suggested that premature intestinal degradation of TDF into TFV may limit the efficiency of the prodrug approach. (Barditch-crovo et al., 2001); (Heimbach et al., 2003); (Roux et al., 2013); (Van Gelder et al., 1999); (Van Gelder et al., 2000); (Van Gelder, 2000); (Gelder et al., 2002) In this study, we further explored the intraluminal stability of the ester prodrug using biorelevant approaches, including in vitro as well as in vivo methods. In a first set of experiments, the in vitro stability of TDF in simulated and human intestinal fluids was determined; subsequently, the in vivo behavior was explored in humans. (Borde et al., 2012)

\subsection{In vitro studies}

\subsubsection{Simulated intestinal fluids}

As suggested by United States Pharmacopoeia 2009 and similar to Borde et al., FaSSIF was supplemented with $10 \mathrm{mg}$ pancreatin per $\mathrm{mL}$ to explore the degradation of ester prodrugs. (Borde et al., 2012); (“U.S. Pharmacopeial Convention," 2009) When TDF was spiked in this solvent system, almost all TDF was degraded within 60 min resulting in the formation of mono-ester intermediate and tenofovir (figure 2). For the fed state, FeSSIF was supplemented with $10 \mathrm{mg}$ pancreatin per $\mathrm{mL}$. The $\mathrm{pH}$ of FeSSIF was not influenced by the addition of pancreatin powder. When adding TDF to this mixture, TDF slowly degraded, resulting in the formation of the mono-ester intermediate; however, no formation of tenofovir could be observed (figure 2), suggesting less chemical and/or enzymatic degradation in fed state conditions. This reduced degradation might be attributed to the lower $\mathrm{pH}$ of FeSSIF (5.0 versus 6.5 in FaSSIF) and/or possible increased micellar entrapment of the hydrophobic TDF $\left(\log D_{5.5}=2.29\right)$ in TDF which may result in a shielding effect of the micelles.

\subsubsection{Human intestinal fluids}

Preliminary experiments showed no degradation of TDF in human gastric fluids obtained in both fasted and fed state conditions; therefore stability was only studied in human intestinal fluids obtained in the 
fasted (FaHIF) as well as the fed state (FeHIF). Upon addition of TDF to FaHIF, TDF converted rapidly to mono-ester and TFV, resulting in complete degradation within one hour (figure 3). From a qualitative point-of-view, the observed TDF degradation in FaSSIF supplemented with pancreatin (figure 2) mimicked TDF degradation in FaHIF. In line with Borde et al. (Borde et al., 2012), however, the degradation rate was higher in human versus simulated fluids. Upon addition of TDF to FeHIF, a slow decrease of the TDF concentration was observed, resulting in the formation of the mono-ester intermediate but not TFV. Similar as for TDF in FeSSIF, this slower degradation can be due to (1) lower pH (5.5 versus 7.6) and (2) micellar entrapment. In addition, food-induced inhibition of enzymatic degradation may result in a higher stability of the prodrug. Similar to the fasted state, an underestimation of degradation was observed in pancreatin supplemented FeSSIF compared to FeHIF; however, the qualitative profile was the same, i.e. the formation of mono-ester intermediate but no tenofovir.

\subsection{In vivo study}

In a next step, we wanted to explore whether these in vitro results are representative for the in vivo situation. To the best of our knowledge, no intraluminal in vivo data is available about the intestinal behavior of ester prodrug. Therefore, a clinical study was performed in which one tablet of TDF (Viread ${ }^{\circledR}$, $300 \mathrm{mg}$ ) was given to healthy volunteers in the fasted as well as the fed state. Subsequently, intestinal fluids from the duodenum were aspirated and analyzed. In parallel to the intestinal sampling, blood samples were collected to link the intestinal behavior of TDF to the appearance of tenofovir in the systemic circulation. A similar approach was already successfully applied to investigate the intestinal behavior of fosamprenavir and posaconazole. (Brouwers et al., 2006); (Walravens et al., 2011)

\subsubsection{Plasma concentration-time profiles}

Figure 4 shows the mean plasma concentration of TFV as a function of time after oral administration of one Viread ${ }^{\circledR}$ tablet containing 245 mg of TDF in fasted and fed state conditions. In plasma, no prodrug nor mono-ester intermediate could be observed (data not shown), suggesting quantitative degradation of TDF during absorption and/or first pass metabolism. In the fasted state, absorption appeared to be very fast 
with a maximum plasma concentration of $978 \pm 183 \mathrm{nM}$ obtained after $28 \pm 5 \mathrm{~min}$. The maximum plasma concentration of TFV in the fed state was observed after $132 \pm 32 \mathrm{~min}$, with a lower $\mathrm{C}_{\max }$ of $711 \pm 162 \mathrm{nM}$ as compared to fasted state conditions. AUC $0-8 \mathrm{~h}$ and $\mathrm{C}_{\max }$ did not significantly differ between fasted $(151 \pm 208 \mu \mathrm{M} \cdot \min ; 978 \pm 183 \mathrm{nM})$ and fed $(148 \pm 394 \mu \mathrm{M} \cdot \mathrm{min} ; 771 \pm 162 \mathrm{nM})$ state conditions; however, $t_{\max }$ was significantly increased in fed state conditions. While the increase in $t_{\max }$ was in line with literature, our study did not reveal a positive food effect on the bioavailability of TFV. (Barditch-crovo et al., 2001) It should be mentioned, however, that the positive food effect reported in literature ( $25 \%$ bioavailability in the fasted state versus $40 \%$ in the fed state) was demonstrated after a daily treatment of 23 days, which makes it difficult to compare with our single dose study. (Barditch-crovo et al., 2001) To further investigate the difference in concentration-time profile between the fasted and fed state, the intraluminal behavior of TDF was assessed by the simultaneous collection of intestinal fluid samples.

\subsubsection{Intestinal concentration-time profiles}

For the collection of the intestinal fluids, a unique sampling method was used whereby one double-lumen polyvinyl catheter was placed via the nose into the duodenum. After the placement of the catheter and the intake of TDF, intestinal fluids were collected and analyzed for TDF, mono-ester intermediate and TFV.

\subsubsection{Fasted state condition}

The concentrations of TDF, mono-ester intermediate and TFV observed in the duodenum are depicted in figure 5 and confirm that rapid degradation is taking place in the intraluminal environment. In the fasted state condition, the mean maximum concentration of TDF $(163 \pm 71 \mu \mathrm{M})$ was reached $24 \pm 4 \mathrm{~min}$ after the intake of the drug. Similar to the in vitro studies (figures 2 and 3), degradation resulted in the formation of both the mono-ester intermediate and tenofovir. The mono-ester intermediate reached a mean maximum concentration of $193 \pm 63 \mu \mathrm{M} 33 \pm 9 \mathrm{~min}$ after intake of the drug. The higher concentration of the mono-ester intermediate compared to the prodrug can probably be attributed to the dual fate of TDF, i.e. the prodrug may be (1) degraded to its mono-ester intermediate, and (2) rapidly absorbed in the nonionized state $\left(\log D_{7.4}=2.65\right)$. Rapid absorption of TDF is confirmed by the short $t_{\max }$ observed for TFV in 
the plasma profiles (figure 4), which corresponds to the relatively short $t_{\max }$ of TDF in intestinal samples. In contrast, the intermediate is not expected to be absorbed due to its negative charge $\left(\log D_{7.4}=-1.54\right)$. In the fasted state, the mean $\mathrm{pH}$ of the intestinal fluids was $6.65 \pm 0.52$ which is in agreement with reported data in the literature (pH between 6.3 and 7.5). (Augustijns et al., 2013) Based on the findings that ester prodrugs are exponentially more sensitive to chemical hydrolysis at a $\mathrm{pH}>4$, chemical degradation cannot be neglected. (Lung-Chi et al., 2001) Also the active compound TFV could be detected in the intestinal samples demonstrating phosphodiesterase activity; no clear $\mathrm{C}_{\max }$ could be observed, with an average concentration fluctuating around $75 \mu \mathrm{M}$.

\subsubsection{Fed state condition}

TDF, the mono-ester intermediate and TFV were also detected in the intestinal samples collected in fed state conditions (figure 6). The combination with food induced a delay in gastric emptying, resulting in increased $t_{\max }$ values and smoother profiles for TDF and its degradation compounds. The concentration of TDF reached its mean maximum concentration $(210 \pm 26 \mu \mathrm{M}) 60 \pm 7.3 \mathrm{~min}$ after drug intake. This differs significantly $(p<0.05)$ from $t_{\max }$ of TDF observed in the fasted state condition $(24 \pm 4 \mathrm{~min})$. The mean maximum concentration of the mono-ester intermediate amounted to $339 \pm 40 \mu \mathrm{M}$, and was reached $84 \pm 14 \mathrm{~min}$ after intake of the tablet. The AUC $0-4 \mathrm{~h}$ of TDF and the mono-ester were both significantly higher in fed versus fasted state. The 3 -fold increase in AUC $0-4$ h of TDF (fed: $13.9 \pm 2.1 \mu \mathrm{M} \cdot \mathrm{min}$ versus fasted: 4.1 $\pm 1.5 \mu \mathrm{M} \cdot \mathrm{min})$ suggests a slower absorption of TDF in fed state conditions. A food-induced delay in gastric emptying and reduction of the absorption rate is reflected in the plasma concentration-time profiles for TFV (figure 4). For TFV, average concentrations fluctuated around $12 \mu \mathrm{M}$; the concentration is much lower compared to the fasted state condition indicating a higher intestinal stability of the monoester intermediate in the fed state.

When considering the ratio of mono-ester intermediate to prodrug, it appears that there is relatively more mono-ester intermediate present in fasted state conditions than in fed state conditions (figure 7). These results indicate slower degradation of TDF in combination with a reduced rate of absorption in postprandial conditions. 
Interestingly, the higher intestinal concentrations of TDF in fed state conditions are not accompanied by higher concentrations in the plasma-concentration profile (figure 4), which suggests that the concentrations measured do not reflect the driving force for absorption. It has been mentioned before that micellar entrapment of the prodrug may occur in view of the relatively high lipophilicity of the ester prodrug. Dialysis experiments were performed to explore the association of TDF with micelles; unfortunately, degradation in the media used prevented us to estimate micellar entrapment.

Compared to the observed in vitro degradation profile of TDF, the clinical study confirmed the faster conversion of TDF to mono-ester intermediate in fasted versus fed state conditions. In fasted state conditions, further degradation to TFV was observed both in vitro and in vivo. In fed state conditions, however, the formation of intraluminal TFV in vivo (albeit significantly less than in fasted state) could not be simulated in vitro. 


\section{CONCLUSION}

The ester prodrug TDF, which has been designed to overcome permeability issues of tenofovir, has a relatively low bioavailability of $25 \%$ in man. In the present study we explored whether the limited efficiency of the prodrug is due to premature degradation in the intestinal tract. Based on in vitro studies performed in biorelevant media, the following conclusions could be made: (1) TDF does not degrade in the stomach due to the absence of esterases (enzymatic stability) and the acidic pH (chemical stability), (2) in fasted state conditions, TDF is rapidly converted to mono-ester intermediate and TFV, and (3) in fed state conditions, the degradation rate of TDF is reduced compared to the fasted state. The clinical study confirmed extensive intraluminal degradation of TDF in healthy volunteers. Similar to the in vitro experiments, the ester prodrug was more stable in the fed state condition compared to the fasted state condition. However, the increased intraluminal stability of the prodrug in postprandial conditions was not reflected in enhanced systemic exposure of TVF. Micellar encapsulation in the intestine may decrease the free concentration as driving force for absorption, and thereby counteract the improved prodrug stability. The results of this study reveal extensive premature degradation of TDF; therefore, tenofovir may benefit from a new prodrug approach. Since fast absorption may (partly) compensate for fast degradation, prodrug selection should be based on (combined) stability and permeability assays. 


\section{FIGURE LEGENDS}

Figure 1: Degradation profile of TDF.

Figure 2: Time-dependent degradation of tenofovir disoproxil fumarate $(\bullet)$ (at initial concentration of $50 \mu \mathrm{M}$ ) to mono-ester intermediate $(\boldsymbol{\square})$ and tenofovir $(\boldsymbol{\Delta})$ in FaSSIF with $10 \mathrm{mg}$ pancreatin/mL $(A)$ and FeSSIF with $10 \mathrm{mg}$ pancreatin/mL (B). Results are expressed as mean $\pm s d(n=3)$.

Figure 3: Time-dependent degradation of tenofovir disoproxil fumarate $(\bullet)$ (at initial concentration of $50 \mu \mathrm{M})$ to mono-ester intermediate $(\boldsymbol{\square})$ and tenofovir $(\boldsymbol{\Delta})$ in FaHIF $(A)$ and FeHIF $(B)$. Results are expressed as mean \pm sd $(n=3)$.

Figure 4: Plasma concentration-time profiles for TFV in the fasted state (A) and fed state (B) after oral administration of a tablet containing 245 mg TDF. Results are expressed as mean \pm sem $(n=5)$.

Figure 5: Duodenal concentration-time profile for TDF (A), mono-ester (B) and TFV (C) in the fasted state after oral administration of a tablet containing $245 \mathrm{mg}$ TDF. Results are expressed as mean $\pm \operatorname{sem}(n=5)$.

Figure 6: Duodenal concentration-time profile for TDF (A), mono-ester (B) and TFV (C) in the fed state after oral administration of a tablet containing $245 \mathrm{mg}$ TDF. Results are expressed as mean $\pm \operatorname{sem}(n=5)$.

Figure 7: Ratio of the concentration of mono-ester intermediate over the concentration of TDF in function of time in fasted $(\bullet)$ and fed $(\mathbf{a})$ state condition after oral administration of a tablet containing $245 \mathrm{mg}$ TDF. Results are expressed as mean \pm sem $(n=5)$. 


\section{FIGURES}

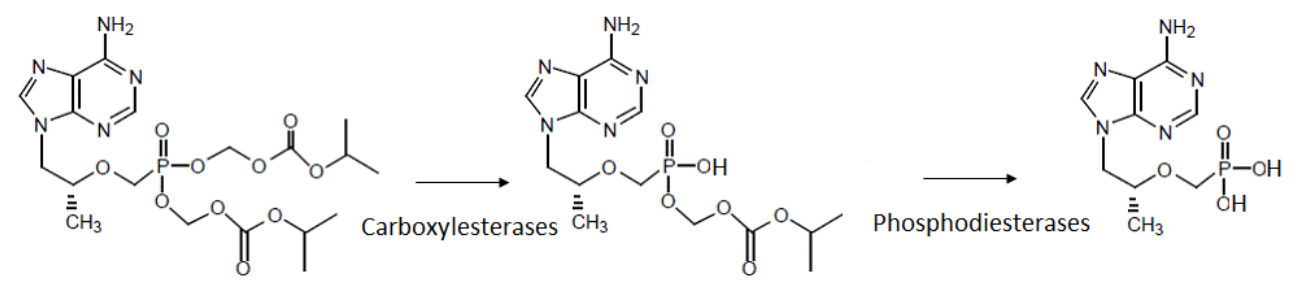

Figure 1

A

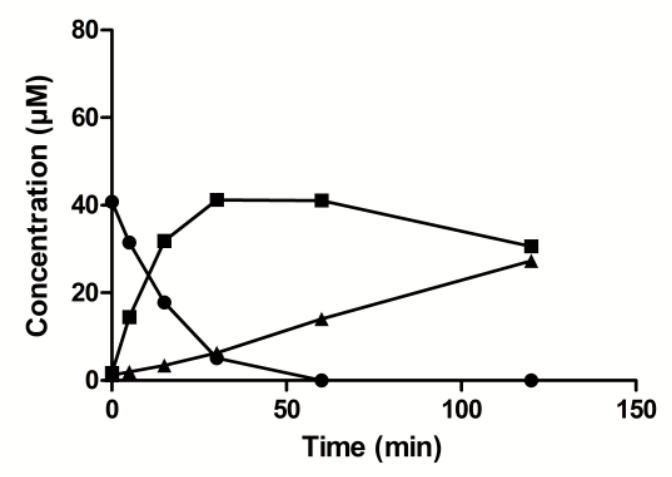

Figure 2

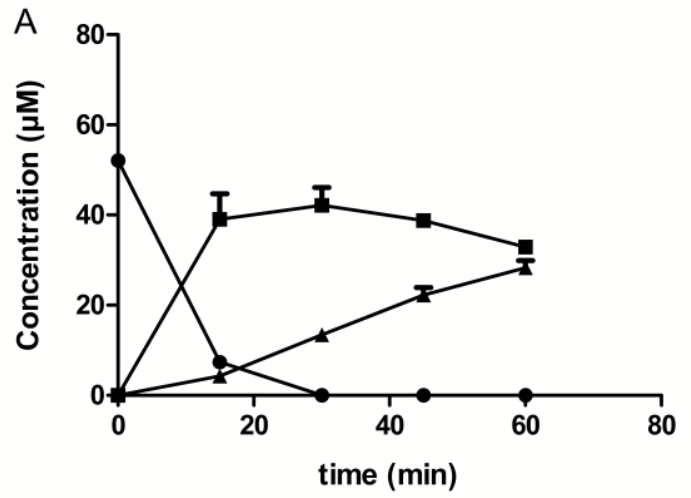

B

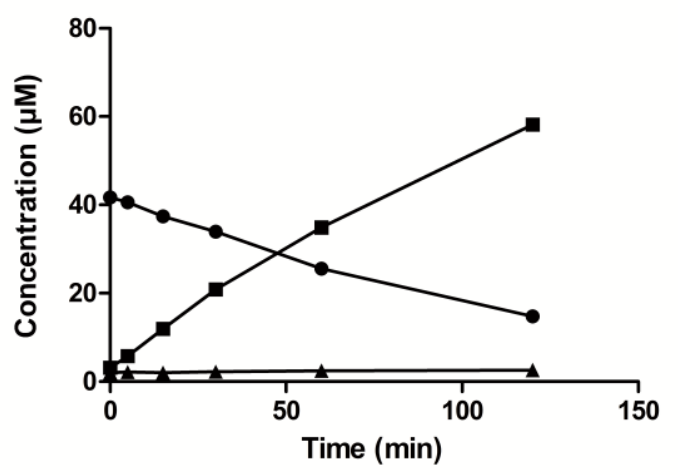

Figure 3

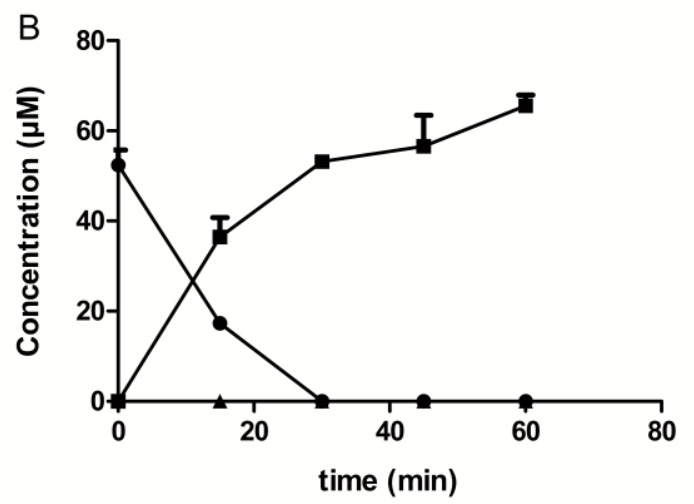



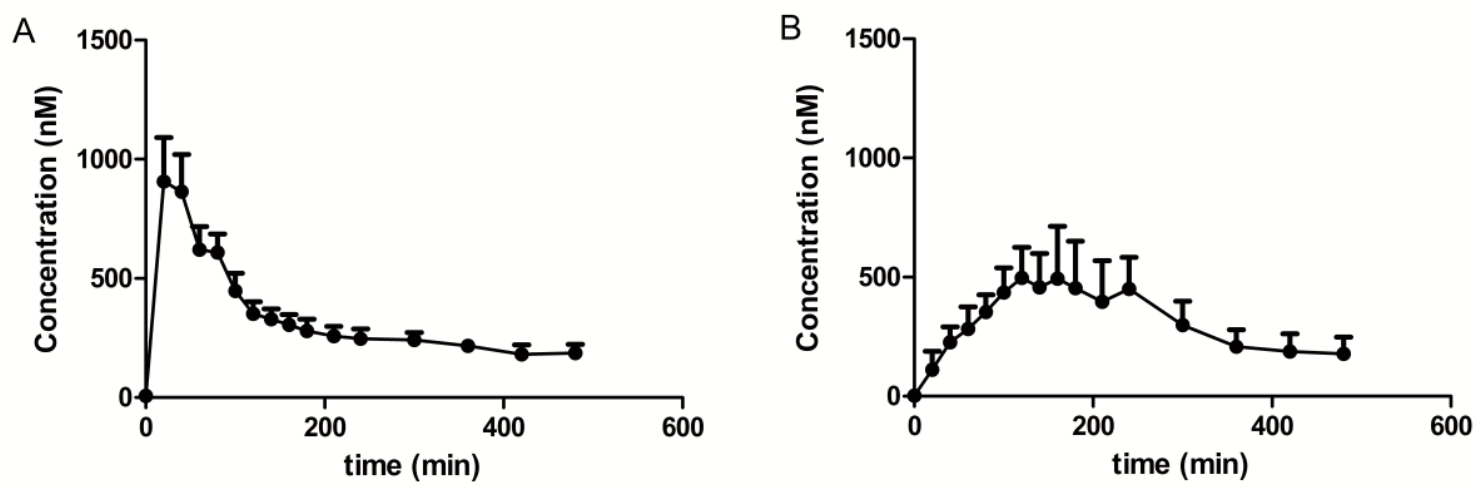

Figure 4
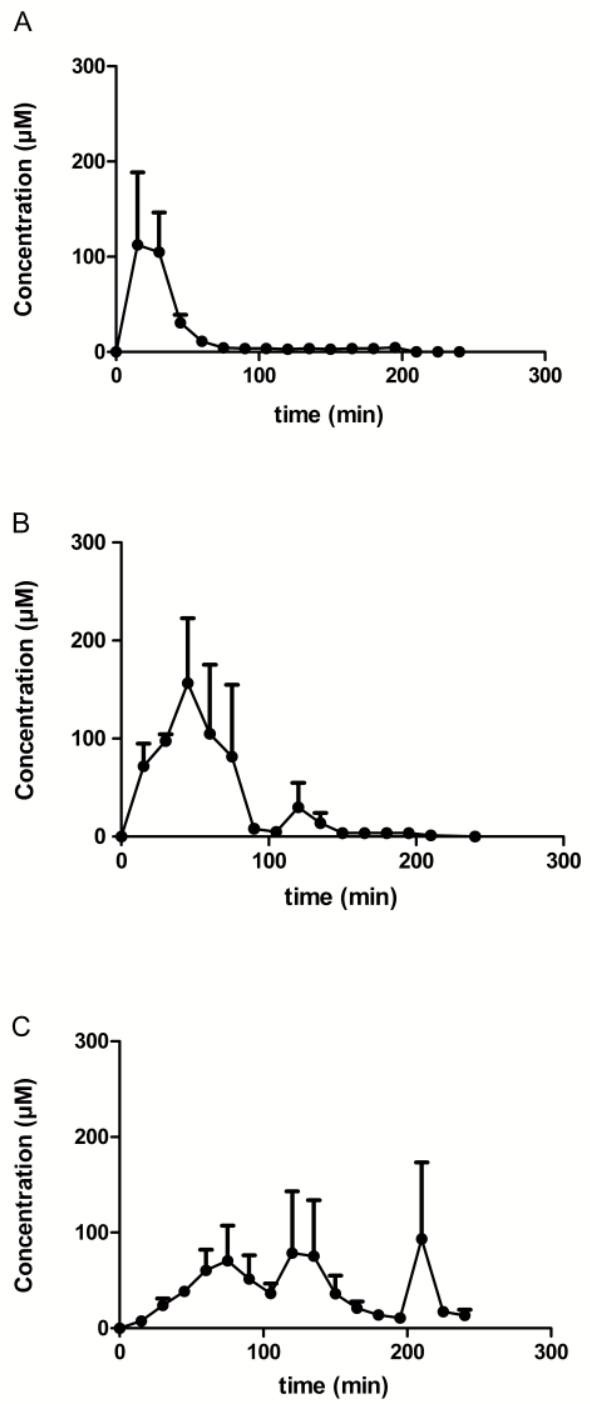

Figure 5 

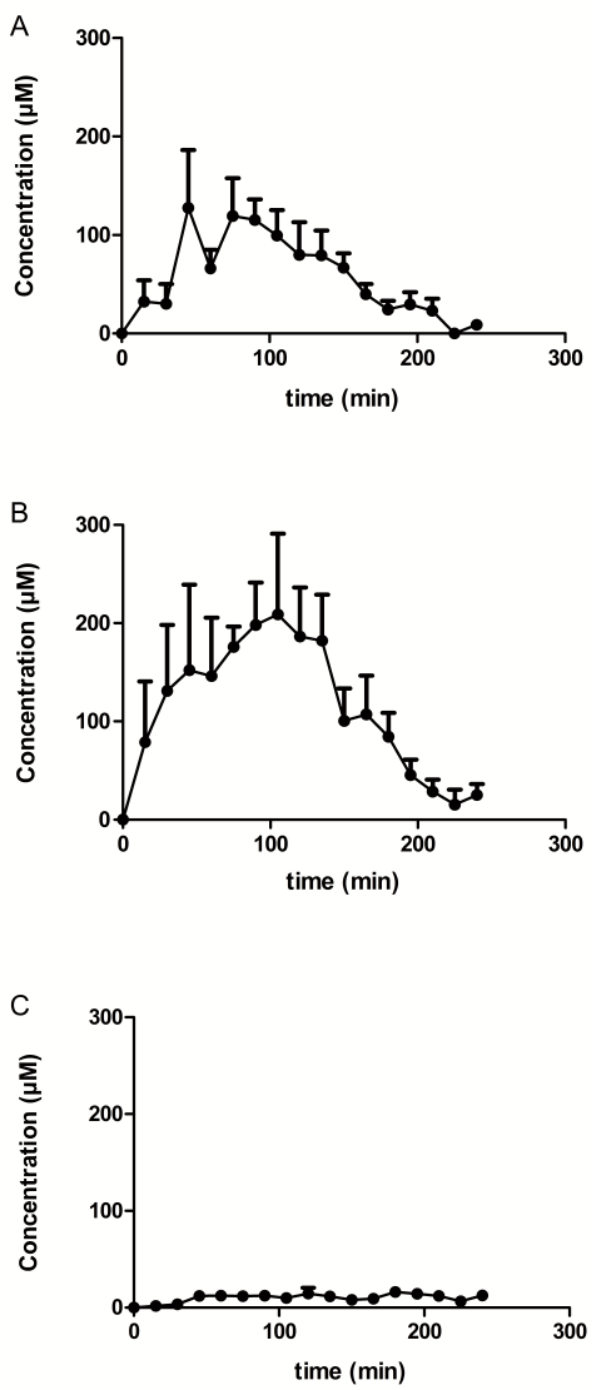

Figure 6

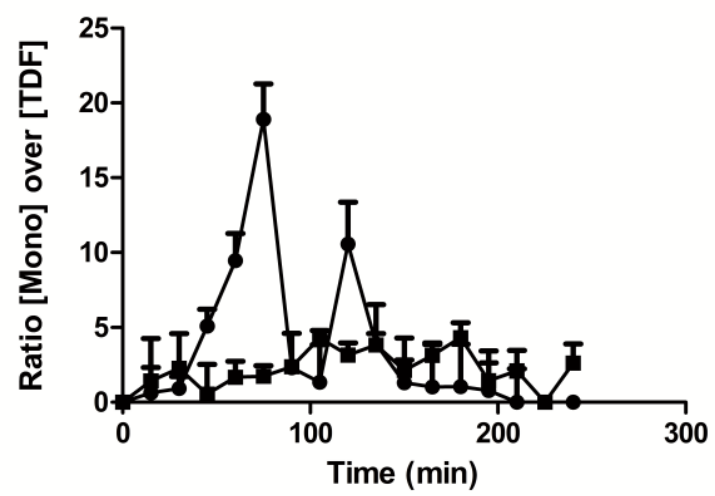

Figure 7 


\section{REFERENCES}

Augustijns, P., Wuyts, B., Hens, B., Annaert, P., Butler, J., Brouwers, J., 2013. A review of drug solubility in human intestinal fluids: Implications for the prediction of oral absorption. Eur. J. Pharm. Sci. 16(57),322-332

Barditch-Crovo, P., Deeks, S.G., Collier, A., Safrin, S., Coakley, D.F., Miller, M., Kearney, B.P., Coleman, R.L., Lamy, P.D., Kahn, J.O., McGowan, I., Lietman, P.S., 2001. Phase I / I trial of the pharmacokinetics, safety, and antiretroviral activity of tenofovir disoproxil fumarate in human immunodeficiency virusinfected adults. Antomicrob Agents Chemother. 45(10), 2733-2739.

Borde, A.S., Karlsson, E.M., Andersson, K., Björhall, K., Lennernäs, H., Abrahamsson, B., 2012. Assessment of enzymatic prodrug stability in human, dog and simulated intestinal fluids. Eur. J. Pharm. Biopharm. 80 (3), 630-637.

Brouwers, J., Augustijns, P., 2014. Resolving intraluminal drug and formulation behavior: Gastrointestinal concentration profiling in humans. Eur. J. Pharm. Sci. 30 (61), 2-10

Brouwers, J., Tack, J., Augustijns, P., 2007. Parallel monitoring of plasma and intraluminal drug concentrations in man after oral administration of fosamprenavir in the fasted and fed state. Pharm. Res. 24 (10), 1862-1869.

Brouwers, J., Tack, J., Lammert, F., Augustijns, P., 2006. Intraluminal drug and formulation behavior and integration in in vitro permeability estimation: a case study with amprenavir. J. Pharm. Sci. 95 (2), 372-383.

Clarysse, S., Brouwers, J., Tack, J., Annaert, P., Augustijns, P., 2011. Intestinal drug solubility estimation based on simulated intestinal fluids: comparison with solubility in human intestinal fluids. Eur. J. Pharm. Sci. 43 (4), 260-269.

Cundy, K.C., Sueoka, C., Lynch, G.R., Griffin, L., Lee, W.A., Shaw, J.P., 1998. Pharmacokinetics and bioavailability of the anti-human immunodeficiency virus nucleotide analog 9-[(R)-2(phosphonomethoxy)propyl]adenine (PMPA) in dogs. Antimicrob. Agents Chemother. 42 (3), 687690.

Fardis, M., Oliyai, R., 2007. Case Study: Tenofovir Disoproxil Fumarate: An Oral Prodrug of Tenofovir. Biotechnology: Pharmaceutical Aspects (V), 1347-1357

Heimbach, T., Oh, D.M., Li, L.Y., Forsberg, M., Savolainen, J., Leppänen, J., Matsunaga, Y., Flynn, G., Fleisher, D., 2003. Absorption rate limit considerations for oral phosphate prodrugs. Pharm. Res. 20 (6), 848-856.

Kearney, B.P., Flaherty, J.F., Shah, J., 2004. Tenofovir disoproxil fumarate: clinical pharmacology and pharmacokinetics. Clin Pharmacokinet. 43(9), 595-612

Lung-Chi, Y., Terrence, D.C., Reza, O., 2001. Degradation Kinetics of Oxycarbonyloxymethyl Prodrugs of Phosphonates in Solution. Pharm. Res. 18(2), 234-237.

Mallants, R., Van Oosterwyck, K., Van Vaeck, L., Mols, R., De Clercq, E., Augustijns, P., 2005. Multidrug resistance-associated protein 2 (MRP2) affects hepatobiliary elimination but not the intestinal 
disposition of tenofovir disoproxil fumarate and its metabolites. Xenobiotica. 35 (10-11), 10551066.

Müller-Lissner, S.A., Fimmel, C.J., Will, N., Müller-Duysing, W., Heinzel, F., Blum, A.L., 1982. Effect of gastric and transpyloric tubes on gastric emptying and duodenogastric reflux. Gastroenterology 83 (6), 1276-1279.

Naesens, L., Balzarini, J., De Clercq, E., 1992. Acyclic adenine nucleoside phosphonates in plasma determined by high-performance liquid chromatography with fluorescence detection. Clin. Chem. 38 (4), 480-485.

Naesens, L., Bischofberger, N., Augustijns, P., Annaert, P., Van den Mooter, G., Arimilli, M.N., Kim, C.U., De Clercq, E., 1998. Antiretroviral efficacy and pharmacokinetics of oral bis(isopropyloxycarbonyloxymethyl)-9-(2-phosphonylmethoxypropyl)adenine in mice. Antimicrob. Agents Chemother. 42 (7), 1568-1573.

Rautio, J., Kumpulainen, H., Heimbach, T., Oliyai R, Oh, D., Järvinen, T., Savolainen, J., 2008. Prodrugs: design and clinical applications. Nat. Rev. Drug Discov. 7(3), 255-270.

Roux, L., Priet, S., Payrot, N., Weck, C., Fournier, M., Zoulim, F., Balzarini, J., Canard, B., Alvarez, K., 2013. Ester prodrugs of acyclic nucleoside thiophosphonates compared to phosphonates: Synthesis, antiviral activity and decomposition study. Eur. J. Med. Chem. 63, 869-881.

Sparidans, R.W., Crommentuyn, K.M.L., Schellens, J.H.M., 2003. Liquid chromatographic assay for the antiviral nucleotide analogue tenofovir in plasma using derivatization with chloroacetaldehyde. J Chromatogr B Analyt Technol Biomed Life Sci. 791(1-2), 227-233.

U.S. Pharmacopeial Convention, 2009. URL http://www.usp.org/ (accessed 1.19.15).

Van Gelder, J., Deferme, S., Annaert, P., Naesens, L., De Clercq, E., Van den Mooter, G., Kinget, R., Augustijns, P., 2000. Increased Absorption of the Antiviral Ester Prodrug Tenofovir Disoproxil in Rat Ileum by Inhibiting Its Intestinal Metabolism. Drug Metab Dispos. 28 (12), 1394-1396.

Van Gelder, J., Anneart, P., Naesens, L., De Clercq, E., Van den Mooter, G., Kignet, R., Augustijns, P., 1999. Inhibition of intestinal metabolism of the antiviral ester prodrug bis(POC)-PMPA by nature-identical fruit extracts as a strategy to enhance its oral absorption: An in vitro study. Pharm Res. 16 (7), 103540.

Van Gelder, J., Deferme, S., Naesens, L., De Clercq, E., Van den Mooter, G., Kinget, R., Augustijns, P., 2002. Intestinal absorption enhancement of the ester prodrug tenofovir disoproxil fumarate through modulation of the biochemical barrier by defined ester mixtures. Drug Metab Dispos.30 (8), 924930.

Van Gelder, J., Shafiee, M., De Clercq, E., Penninckx, F., Van den Mooter, G., Kinget, R., Augustijns, P., 2000. Species-dependent and site-specific intestinal metabolism of ester prodrugs. Int. J. Pharm. 205 (1-2), 93-100.

Walravens, J., Brouwers, J., Spriet, I., Tack, J., Annaert, P., Augustijns, P., 2011. Effect of pH and comedication on gastrointestinal absorption of posaconazole: monitoring of intraluminal and plasma drug concentrations. Clin. Pharmacokinet. 50 (11), 725-734. 